\section{A5.18 IMPACT OF RITUXIMAB ON SYNOVIAL GENE EXPRESSION}

doi:10.1136/annrheumdis-2013-203219.18

Yasser M El-Sherbiny, Sarah Churchman, Paul Emery, Edward M Vital, Frederique Ponchel. Division of Rheumatic and Musculoskeletal Disease, Leeds Institute of Molecular Medicine, St James's University Hospital, Beckett Street, Leeds, UK

Introduction Quality and duration of rituximab therapy response in rheumatoid arthritis have not been totally explained. In the synovium, three studies have indicated a relationship between higher plasma cell numbers at baseline and worse, or shorter lasting responses [1-3]. In this study we measured synovial expression of genes involved in B-cell biology, cell survival, cell trafficking and inflammation.

Methodology Synovial arthroscopic biopsies were collected and snap frozen from patients at baseline and 26 weeks after rituximab. Immunohistochemistry was performed for CD3, CD19, CD20cy, CD138 and CD68 and scored for positive cells $/ \mathrm{mm}^{2}$ using digital image analysis. The remainder of the tissue was used for RNA extraction. A 48 gene custom Taqman array was designed, including genes for cell lineage markers (CD19, CD20, CD3, CD138 \& CD68), BAFF-APRIL system, immunoglobulins, cytokines, chemokines and adhesion molecules or cell trafficking. Informative data are available from 32 biopsies pre-Rituximab treatment and 23 post-treatment. Unsupervised clustering was performed to all gene expression to all patients pre and post rituximab.

Results Clustering of gene expressions revealed 3 main groups pre-rituxmab synovial biopsies, a definite cluster of B cell/survival factors signature associated low expression other genes synovial biopsies was associated with a higher disease activity (DAS28) at baseline and after treatment compared to the other two groups pooled together. This resulted in a lower rate of EULAR Good response.

Comparing EULAR responders and non-responders, there were trends to higher baseline CD20 gene expression in non-responders $(p=0.079)$ and greater reduction in CXCL13 ( $p=0.066)$ and MMP $(p=0.024)$ in responders.

Lower expression of ICAM ( $p=0.021)$, FGF $(p=0.044)$, CD20 $(p=0.055)$ and $p 53(p=0.025)$ and higher expression of APRIL $(p=0.029)$ at baseline was associated with normalisation of CRP after therapy. Furthermore, these patients also showed a significantly greater reduction in expression of CD4, CD55, CD68, CXCL12, EGF, FGF, ICAM, PECAM, STAT5, TGF-beta, APRIL and BAFF (all $p<0.05$ ).

Conclusions Results point to key differences in synovial gene expression in patients with clinical response to rituximab, obviously in relation with genes involved in cell trafficking and survival.

\section{References}

1. Teng et al, $A \& R$ (2008) 56(12).

2. Thurlings et al, ARD (2008) 67

3. Vital et al, EULAR 2012

\section{A5.19 IN AFRICAN PATIENTS WITH RHEUMATOID ARTHRITIS ACPA RECOGNISE CITRULLINATED FIBRINOGEN AND THE IMMUNODOMINANT EPITOPES BORNE BY THE FIBRIN PEPTIDES a36-50 AND $\beta 60-74$, LIKE IN CAUCASIANS}

doi:10.1136/annrheumdis-2013-203219.19

${ }^{1,2} \mathrm{~L}$ Nogueira, ${ }^{1} \mathrm{M}$ Cornillet, ${ }^{3} \mathrm{M}$ Singwe-Ngandeu, ${ }^{4} \mathrm{~S}$ Viatte, ${ }^{5,6} \mathrm{~S}$ Bas, ${ }^{5,7} \mathrm{C}$ Gabay, ${ }^{1,2} \mathrm{G}$ Serre. 'Laboratory of "Epidermis Differentiation and Rheumatoid Autoimmunity", UMR CNRS 5165, INSERM U 1056, Toulouse III University; 'Laboratory of Cell Biology and Cytology, University Hospital of Toulouse; Toulouse, France; ${ }^{3}$ Unit of Rheumatology, School of Medicine, University of Yaoundé, Cameroun; ${ }^{4}$ Arthritis Research, UK Epidemiology Unit, University of Manchester; ${ }^{5}$ Division of Rheumatology, University Hospitals of Geneva; ${ }^{6}$ Department of Genetics and Laboratory Medicine; 'Department of Pathology \& Immunology; University of Geneva, School of Medicine, Genève, Suisse
Background and Objectives Although most studies concerning the Rheumatoid Arthritis (RA)-specific antibodies to citrullinated proteins (ACPA) have been performed in populations of Caucasian ancestry, anti-CCP2 antibodies were demonstrated to be also good markers for RA in African patients. In one cohort from Cameroon, frequency of the HLA-DRB1 shared epitope (SE) alleles, although higher in RA patients than in controls, was lower than in Caucasian patients. Furthermore, known Caucasian non-HLA susceptibility genes did not confer the same risk in Africans.

The purposes of the present study were, first, to analyse in African patients with RA the autoantibodies to citrullinated human fibrinogen (AhFibA), then, to investigate to what extent the two citrullinated fibrin peptides $\alpha 36-50$ and $\beta 60-74$, which bear the immunodominant epitopes, were recognised by the sera of these patients.

Methods AhFibA and autoantibodies to $\alpha 36-50$ and $\beta 60-74$ were measured by ELISA in the serum of 56 consecutive RA patients with established disease from the Rheumatology unit of Yaoundé, Cameroon. 101 patients with other rheumatic diseases or healthy individuals were used as controls.

Results Using the 95\%-specificity thresholds previously defined with a French cohort, $73 \%$ of the RA sera from African patients were positive for AhFibA versus $83 \%$ in French patients. The anti- $\beta 60-74$ and anti- $\alpha 36-50$ autoantibodies were detected in $41 / 56$ (73\%) and 25/56 (45\%) African RA sera versus $71 \%$ and $51 \%$ in French RA patients, respectively. Moreover, 38/41 (93\%) of the AhFibA-positive sera recognised either $\alpha 36-50$ and/or $\beta 60-74$, as previously observed in French patients. Finally, the mean AhFibA $(\mathrm{OD}=1.28)$ and anti- $\beta 60-74(\mathrm{OD}=1.32)$ titers were significantly higher in patients with at least one copy of the HLA-DRB1 SE $(17 / 56,30 \%)$ than in those without SE $(\mathrm{OD}=0.64$ and 0.60 , respectively; $p<0.005$ and $p<0.02)$, while the titer of anti- $\alpha 36-50$ autoantibodies did not differ.

Conclusions AhFibA are markers for RA also in African patients. In these patients, autoantibodies to $\alpha 36-50$ and $\beta 60-74$ peptides are present in proportions similar to those found in Caucasians. Like in Caucasians, they account for almost all the reactivity to fibrinogen. HLA-DRB1 SE alleles, although less common among African RA patients, are associated with higher titres of AhFibA and of autoantibodies to $\beta 60-74$, suggesting that SE alleles partly control the AhFibA production, besides still unknown factors. This study shows that even in different genetic backgrounds the ACPA response is a hallmark of RA, and strongly suggests that its fine specificity to citrullinated fibrin is identical in Caucasians and Africans.

\section{A5.20 INTRAVENOUS IMMUNOGLOBULIN INDUCES FUNCTIONNAL SILENCING IN HUMAN B LYMPHOCYTES}

doi:10.1136/annrheumdis-2013-203219.20

Sophie Hillion, Jean-François Seité, Carole Goutsmedt, Pierre Youinou, Jacques-Olivier Pers. EA2216 Immunology \& Pathology, Brest University and European University of Brittany, Brest, France

Purpose/Objective Inappropriate B cell receptor (BCR) signalling contributes to the activation of auto-antibody-producing $B$ cells in many autoimmune diseases. These diseases results from a break in B-cell tolerance which is normally maintained by the mechanisms of anergy, receptor editing and deletion. We previously showed that IVIg modulates many B cell responses through a sialic acid-CD22 lectin interaction (Séité, Blood 2010, Séité, J Autoimmunity, 2011). Here, we reasoned that IVIg could modulate B-cell fate and may render autoreactive $B$ cells tolerant through induction of a functional unresponsiveness response to BCR stimulation.

Material and Methods Tonsilar B-cells were stimulated in the presence of different fractions of IVIg. We analysed subsequent responses to BCR stimulation by confocal microscopy, quantitative RT-PCR, FACS and Western-blot analysis. 
Results First, we observed that up-regulation of costimulatory molecules CD86, CD80 and CD40 induced by BCR cross-linking was down-regulated after IVIg incubation and was associated with a massive reduction of tyrosine phosphorylation. Low mobilisation of intracellular Calcium, which is a hallmark of anergy, was also observed in IVIg-treated B cells. Next, we observed that following BCR stimulation, IVIg blocks BCR aggregation within lipid rafts and increases its internalisation. Consequently, BCR stimulation is not achievable and results in a low activation of the PI3K-Akt signalling pathway. Finally, we demonstrated that IVIg down-regulates $\mathrm{NF \kappa B}$ activation and promotes NFAT transcription factors entry in the nucleus. These findings demonstrated that IVIg induces a selective transcriptional programme, allowing nuclear signals to be independently activated, leading to alternative B cell fates.

Conclusions Our data suggest that IVIg could induce B cells to adopt a state that results in a functional silencing, also called anergy. Our findings provide insights into the effectiveness of IVIg in treating pathologies associated with the loss of B cell tolerance. We also describe a new model to explore the complexity of positive versus negative selection in human B cells.

\section{A5.21 LACK OF IL-27R SIGNALING LEADS TO AN INTRINSIC B CELL DEFECT AND PROTECTION AGAINST CIA}

doi:10.1136/annrheumdis-2013-203219.21

1,20dilia BJ Corneth, 1,2Patrick S Asmawidjaja, ${ }^{3}$ Laurens P Kil, ${ }^{1,2}$ Anne-Marie C Mus, 1,2Franka Luk, ${ }^{1,2}$ Sandra MJ Paulissen, ${ }^{3}$ Rudi W Hendriks, ${ }^{1,2}$ Erik Lubberts. 'Departments of Rheumatology; ${ }^{2}$ Immunology; ${ }^{3}$ Pulmonary Medicine, Erasmus MC, University Medical Center, Rotterdam, The Netherlands

Introduction The IL-27 receptor (IL-27R) is expressed on naïve $\mathrm{T}$ helper cells. Signaling induces Th1 differentiation and IL-10 production and inhibits Th17 differentiation. In addition, the IL-27R is also expressed on B cells, where it is linked to B cell proliferation and IgG2a class switch recombination. However, its role is not fully understood. We therefore aim to determine the role of IL-27R signalling on B cells, both in $\mathrm{T}$ cell independent and in $\mathrm{T}$ cell dependent immune responses.

Methods To determine the effect of IL-27 on B cells, we isolated naïve $B$ cells from wild type mice and cultured them in the absence or presence of rIL-27. T cell dependent responses were investigated using the collagen induced arthritis (CIA) model in wild type and IL-27R deficient (WSX-1 deficient) mice. T cell independent B cell responses were investigated using the TNP-Ficoll immunisation model.

Results Naïve wild type B cells cultured in the presence of rIL-27 showed increased proliferation and expression of activation markers. Next, we investigated the role of IL-27R signalling in vivo in CIA. Both the incidence and severity of CIA were significantly lower in IL-27R deficient mice. Splenic germinal centre B cells were decreased in these mice. In addition, both auto-reactive IgM and IgG2a antibody levels were significantly decreased. These data suggest an impairment of B cell immunity in IL-27R deficient mice. However, CIA also depends on $\mathrm{T}$ cells. To determine whether the impairment of B cell immunity in IL-27R deficient mice was B cell intrinsic, we used the T cell independent TNP-Ficoll immunisation model. Wild type and IL-27R deficient mice were immunised with TNP-Ficoll i.p. and sacrificed seven days later. IL-27R deficient mice had fewer splenic IgM and IgG3 plasma cells and lower serum TNP specific IgM and IgG3 antibody levels.

Conclusions Here we show that IL-27R signalling on B cells is essential for proliferation and activation of B cells. Impaired B cell immunity in IL-27R deficient mice is caused by an intrinsic B cell defect, leading to impaired plasma cell formation and antibody production in both $\mathrm{T}$ cell independent as well as $\mathrm{T}$ cell dependent immune responses.

\section{A5.22 MEMBRANE-BOUND AND SOLUBLE BAFF EXPRESSION BY HUMAN RHEUMATOID FIBROBLAST-LIKE SYNOVIOCYTES IN RESPONSE TO TLR STIMULATION}

doi:10.1136/annrheumdis-2013-203219.22

${ }^{1} \mathrm{NW}$ Kam, ${ }^{2} \mathrm{~A}$ Filer, ${ }^{2} \mathrm{C}$ Buckley, ${ }^{1} \mathrm{M}$ Bombardieri, ${ }^{1} \mathrm{C}$ Pitzalis. 'William Harvey Research Institute, Queen Mary University of London, London, UK; 'Division of Immunity and Infection, College of Medical and Dental Sciences, University of Birmingham, UK

Background and Objectives $B$ cell activating factors of TNF family (BAFF) is associated with the survival and maturation of $B$ cells. BAFF is widely expressed in the rheumatoid arthritis (RA) synovium which is characterised by the presence of synovial niches of autoreactive B cells and sustain in situ autoantibody production. Importantly, B cell niches remain functional in the RA-SCID model in the absence of recirculating cells, suggesting that autocrine mechanisms support ongoing B cell activation in the RA synovium. BAFF exerts its functional role both as a membrane bound protein and in soluble form. Here we investigated whether resident stromal cells in the RA synovium, synovial fibroblasts (RASF), are capable of producing either forms of BAFF and thus contribute to local $B$ cell activation.

Methods mRNA BAFF in RASF stimulated with TLR2, TLR3 and TLR4 ligands was assessed by quantitative Taqman PCR RA dermal fibroblasts (RADF) and osteoarthritis SF (OASF) were used as controls. The cytoplasmic, membrane bound and/or soluble forms of BAFF were investigated by 1) Western blot using total and membrane-enriched protein extracts, 2) flow cytometry, 3) ELISA and 4) immunocytochemistry.

Results In vitro stimulation of TLR3, and to a significantly lesser extent TLR4, but not TLR2 on RASF led to strong induction of BAFF mRNA. In response to TLR3, soluble BAFF was time-dependently released in the supernatant of RASF $(\sim 600 \mathrm{pg} / \mathrm{ml})$ and, to a lesser extent, OASF and RADF. RASF constitutively expressed both cytoplasmic and membrane bound BAFF as demonstrated by WB, FACS and immunocytochemistry which was upregulated upon TLR3 stimulation and was significantly increased as compared to RADF.

Conclusions Here we provide conclusive evidence that SF in the RA synovium are a pivotal source of the B cell survival factor BAFF at both mRNA and protein level. In addition to their significant constitutive expression, RASF can further up-regulate cytoplasmic, membrane-bound and soluble BAFF in response to TLR3 stimulation. Overall, our data strongly support a fundamental role for RASF in sustaining functional B cell activation and antibody production in the inflamed RA synovium.

\section{A5.23 MULTIPARAMETER PHOSPHO-FLOW ANALYSIS OF B CELLS FROM PATIENTS WITH RHEUMATOID ARTHRITIS}

doi:10.1136/annrheumdis-2013-203219.23

'Daniel Szili, ' Judit Pozsgay, 'Zsuzsanna Banko, ${ }^{2}$ Tamas Gati, ${ }^{2,3}$ Gyorgy Nagy, ${ }^{2}$ Bernadett Rojkovich, 'Gabriella Sarmay. 'Department of Immunology, Eötvos Lorand University, Budapest; ${ }^{2}$ Buda Hospital of Hospitaller Brothers of St. John, Budapest; ${ }^{3}$ Department of Rheumatology, Semmelweis University, Budapest, Hungary

Background and Objectives Rheumatoid arthritis (RA) is a common, relapsing autoimmune disease, which affects approximately $1 \%$ of the population worldwide. While the specific molecular events that lead to initiation and onset of RA are not known, an uncontrolled activation of the immune system is considered to be a critical component of the disease. B lymphocytes undoubtedly play a critical role in disease aetiology. Antigen binding to B-cell receptor (BCR) triggers $B$ cell activation, although the threshold of activation can be influenced by other receptors, such as TLR9. TLR9 has received substantial attention as a pathogenic co-stimulator of 